\title{
INVESTIGATION ON TO THE ILLNESS AND DEATH OF ZEBRA AT DHAKA ZOO ALONG WITH ISOLATION AND IDENTIFICATION OF CAUSAL ORGANISM
}

\author{
S. A. Happy, M. Pervin, M. S. Azam¹, A. S. M. Bari and M. A. H. N. A. Khan \\ Department of Pathology, Bangladesh Agricultural University \\ Mymensingh-2202, Bangladesh
}

\begin{abstract}
Zebra is the member of the equide family available at Dhaka Zoo and gradually disappeared due to inadequate health management and varies maladies. The aim of this study was to investigate the cause of illness and death of zebras (Equus burchelli) at Dhaka Zoo. Two zebras died on 10 and 25 January, 2010, one become seriously ill on 15 February, 2010 and was considered as the study samples. The dead animals were necropsized, representative samples were collected in formalin for histopathological examination. Thoracic fluid, cardiac fluid, abdominal fluid and blood from these zebras were also used for isolation of causal bacteria. Blood sample was also collected from the sick zebra and subjected for bacteriological examination. Results of necropsy examination revealed hydrothorax, pneumonia and septicemia. Histopathological findings revealed pulmonary emphysema, interstitial pneumonia, bronchitis, pulmonary thrombosis, exudates in lung alveoli and granulomatous lesions in lungs. Other important findings were hemosiderosis and anthracosis. Based on the cultural, morphological and biochemical tests, Pasteurella sp. was identified. Bacteriological examination revealed that the organism bears similarities with $P$. multocia, P. hemolytica and P. aerogenes but definite bacterial species was not identified. The zebras were collected from Africa and the bacterial nature identified was Pasteurella but definite species could not be identified; could be a Pasteurella available in Africa. Therefore, it needs investigation at other biochemical and genetic level to confirm the bacterial species involved. The reoccurrence of similar disease at later on was also observed in zebra at Dhaka Zoo and responded well to treatment for pasteurellosis; the cause of illness was, therefore, confirmed as Pasteurellosis.
\end{abstract}

Key Words: Zebra, Pasteurellosis, Hemosiderosis, Anthracosis, Dhaka Zoo

\section{INTRODUCTION}

The World Zoo Conservation Strategy emphasizes that the use of a variety of educational techniques, facilities and considerations, together with knowledge, creativity and inventiveness can make zoos highly interesting, attractive and effective places for

\footnotetext{
1 Scientific Officer, Dhaka Zoo, Mirpur-1216, Bangladesh
} 
environmental, conservation and holistic life system education. Today zoological gardens/zoos provide an opportunity to open up a whole new world of curiosity and interest in the natural world and sensitize visitors regarding the value and need for conservation of wildlife (Raghunathan and Nareshwar, 2000). Zoos play a very important role in educating the public about animal conservation. For instance, the signs and posters that zoos put up help make conservation problems more comprehensible (Anon, 2011).

Dhaka Zoo the largest and the national zoo of Bangladesh, it is nicely vegetated and situated. The Dhaka Zoo among eleven others in this country having heritage of the oldest zoo in Bangladesh, has 214 acres of land. It is located at Mirpur-1, $16 \mathrm{~km}$ off from Dhaka zero point and northwest of Dhaka city. Visitor turnout of Dhaka Zoo is about five million annually. The zoo contains many native and non-native animals and wild life. The total number of vertebrate fauna in the zoo is about 2,150 in 192 species. Included in these animals, are about 551 mammals under 64 species, 1,543 birds under 90 species, 73 reptiles under 15 species, and about 104 aquarium fishes under 23 species. To attract visitors besides many fascinating animals, there are 15 tigers, 21 lions, 9 hippopotamus, about 200 monkeys, and 33 pythons. Moreover, some rare and interesting animals such as the rhea, peacock, zebra, elephant, African grey parrot, water buck, impala, emu, baboon, chimpanzee, gayal, black bear, tapir, mandrill, and estuarine crocodile provide additional entertainment to the visitors.

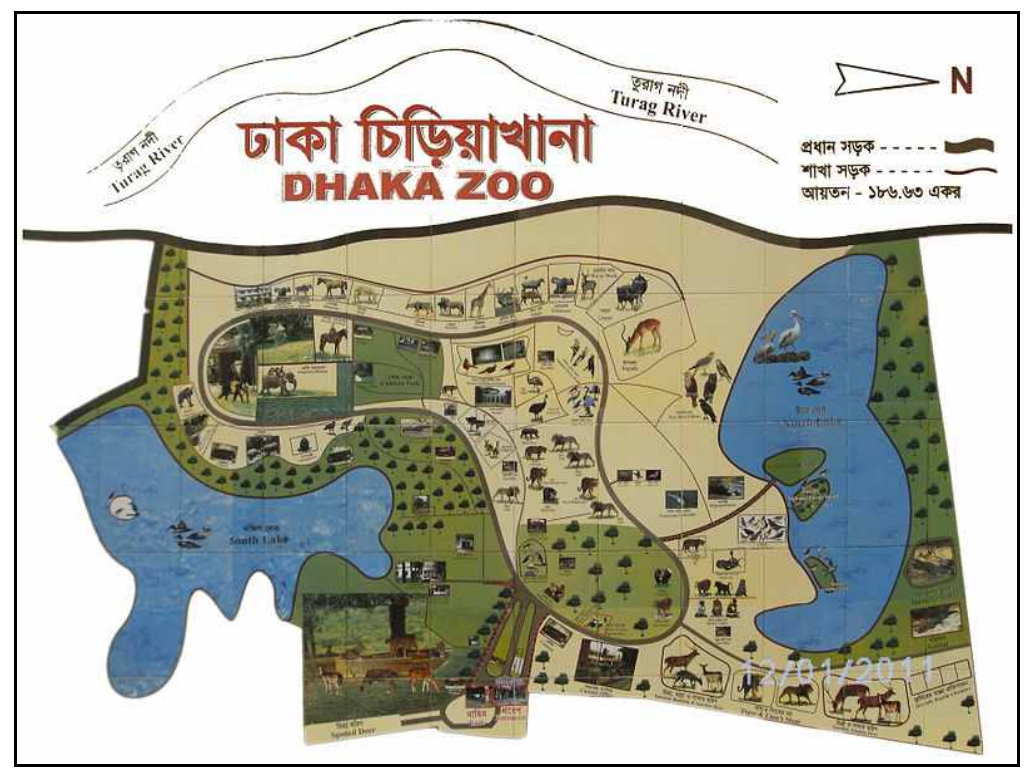

Among the zoo animal's zebras are strikingly beautiful. Zebras are member of the equide family and are a popular subject in art. There are three species of zebra: the Plains Zebra, Grévy's Zebra and the Mountain Zebra. The unique stripes and behaviors of zebras make these among the animals most familiar to people (McClintock, 1976). 
The diseases Pasteurellosis is caused by bacteria with an important role as primary or opportunistic, mainly respiratory, pathogens in domestic and wild animals. Some species of Pasteurellaceae cause severe diseases with high economic losses in commercial animal husbandry and are of great diagnostic concern (Dousse et al., 2009). Pasteurella are commonly isolated from a variety of animal species including cattle, sheep, swine, poultry, dogs, cats, and rabbits, but are rarely isolated from horses and zebra. Out breaks of Pasteurella multocida infection have been reported among horses and donkeys in India (Linda and Schlater, 1989 and Dhand, 2004). Morbidity is higher when animals are herded closely, in poor condition, or exposed to wet conditions. Mortality is nearly $100 \%$ unless the animal is treated very early in the disease; few animals survive once they develop clinical signs (OIE, 2007). Pasteurellosis caused by Pasteurella multocida is reported in zebra (Equus burchelli), kangaroos (Macropus rufus and, ostrich (Struthio camelus camelus) died in Kano Zoo (Okoh, 1980). Pasteurellosis can be transmitted by the bites or scratches of cat and dog or by direct exposure to their secretions (Migliore et al., 2009).

Beside pasteurellosis, anthracosis was also reported in different animals including cattle, horse, dog, lions, leaf monkey and leopard (Jones et al., 1999; Jubb et al., 1993; Murali Manohar et al., 2006 and Sujatha et al., 2007) maintained in the industrial areas and in the coal mines (Jones et al., 1997 and Sujatha et al., 2007). Reports on the occurrence of anthracosis in captive wild animals are sparse. Anthracosis is reported in a number of animal species at Dhaka Zoo (Motu, 2009).

Pathologists at the San Diego Zoo began to notice iron storage in the internal organs of captive lemurs. Hemosiderin was found in liver, spleen, lymph nodes, duodenum, and occasionally other organs (John et al., 1984 and Kristine et al., 2008).

There have been no published report describing diseases of zebra in Bangladesh. This study was, therefore, aimed to investigate the cause(s) of death of zebras at Dhaka Zoo using traditional and biochemical analysis.

\section{MATERIALS AND METHODS}

\section{Samples}

Two dead and two sick zebras from Dhaka Zoo were the materials for this study. The present research work was divided into two phases; histopathological examination of selected tissues and isolation and identification of causal organism.

\section{Necropsy and histopathology}

After necropsy internal organs such as liver, lungs, spleen, kidney, and heart, intestine were collected, and fixed in $10 \%$ neutral buffered formalin. The formalin fixed tissues were processed, sectioned and stained with hematoxiline and eosine (H \& E) stain (Luna et al., 1968). The necropsy examination revealed severe septicemia and consequently thoracic fluid, abdominal fluid cardiac fluid and blood from the dead and sick animals were collected in the sterile test tube and used for isolation and identification of causal bacteria. 


\section{Isolation and identification of causal bacteria}

Impression smears were made from the heart blood onto the clean slide and stained with Gram's staining (Luna et al., 1968). Loop full samples were placed in nutrient broth and streaked onto nutrient agar, blood agar, EMB agar and MacConkey agar plate. The agar plate was incubated overnight at $37^{\circ} \mathrm{C}$ for 24 hours to observed the growth of the organisms. Based on the colony characteristics and appearance, the suspected colonies were then isolated and also stained by Gram's staining and Leishman's staining method (Luna et al., 1968).

\section{Biochemical tests}

Selected colonies were used in different biochemical tests (Carbohydrate fermentation test, MR-VP test, indol test and catalase test) and results were analyzed for the identification of the isolate.

\section{RESULTS}

\section{Necrosy}

The carcass appeared pale, emmaciated and hide bound. The lungs were congested, there were petechiae in the upper respiratory truct and fluid in thoracic cavity. Heart found to contain clotted blood in left ventricle. Gross changes of different tissues of female zebra seen were congested and necrosed lungs and focal necrosis in liver. There were petechial hemorrhages in spleen, coronary fat and pericardium. Pericardial sac and thoracic cavity were filled with clingy serous fluid. The kidney was hemorrhagic and urinary bladder was filled with bloody urine. The mucosa of gastrointestinal tract was hemorrhagic and intense hemorrhagic lesion was seen in duodenal lumen. The tentative cause of death of zebra was considerd as septicemic infection.

\section{Histopathology}

Results of histopathologic examination revealed generalized hemorrhages through out of the body. The lungs were consolidated (Fig. 1A), and inflamed. There was deposition of pink color edematous fluid in the distended and ruptured lungs alveoli (Fig. 1B). Emphysematous lungs (Fig. 1C) and anthracosis (Fig. 1D) were also noticed in the lungs of dead zebra. Deposition of exudates in the bronchial lumen and intravascular coagulation in the vasculature of lungs was seen.

There was lymphoid depletion in spleen (Fig. 2A) and lymph node (Fig. 2B). Deposition of golden brown color pigment in the cytoplasm of macrophages in the spleen (Fig. 2A), lymphnode (Fig. 2B) and lungs was commonly seen.

\section{Causal agent}

The causative organism was grown in nutrient broth and various agar medium. Results of this study showed small white dew drop like colonies on blood agar media Fig. 3A. The colony appeared opaque, round, slightly raised, smooth, shiny grayish-yellow in color, and non adherent. Hemolysis was not seen on blood agar culture. On MacConkey 
agar organism produced small pink color colonies which is characteristics of Pasteurella $s p$. While the organism was grown on EMB agar, did not develop metallic sheen and thus differ from coliform organism.
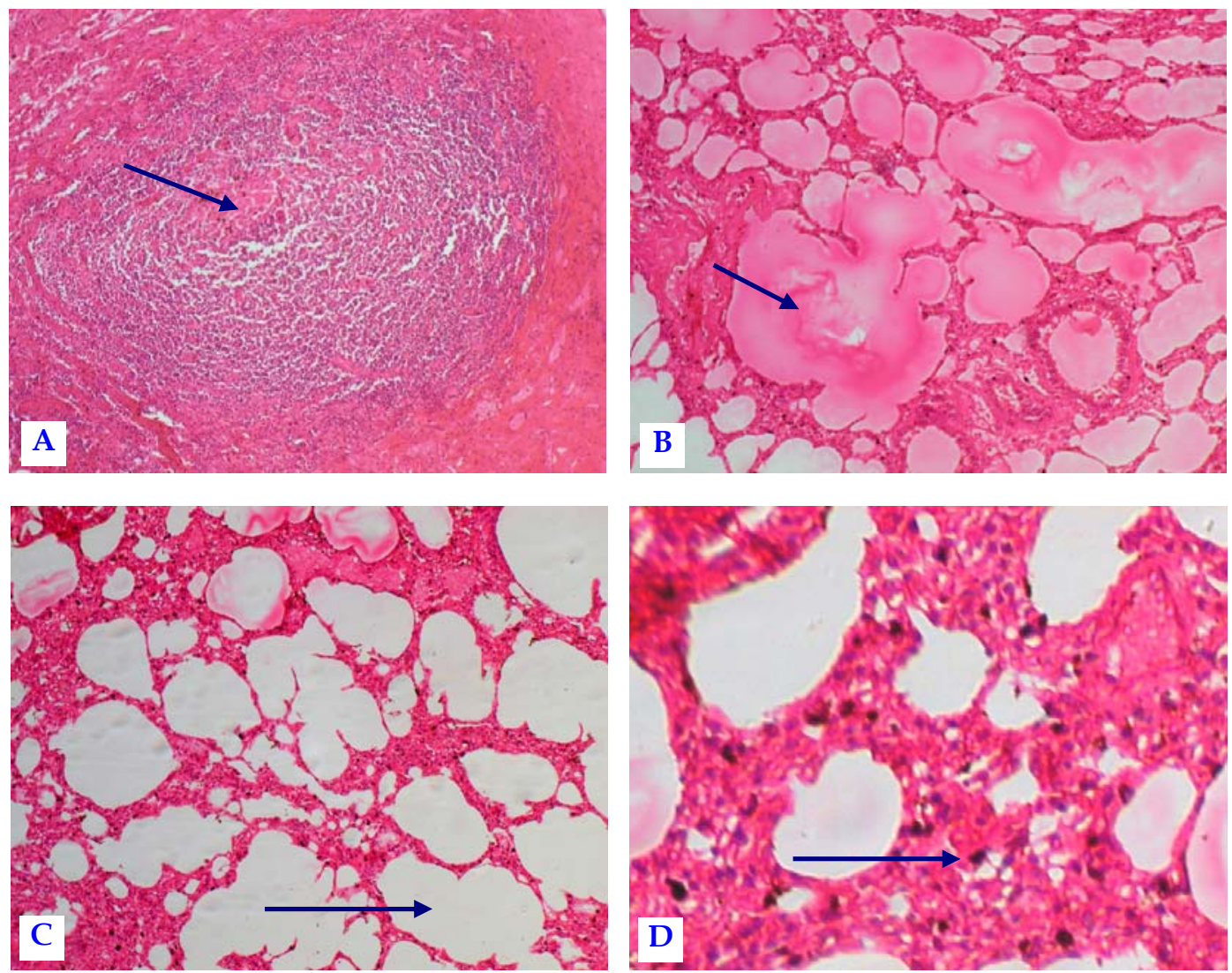

Fig. 1. Section of the lungs of a dead zebra obtained from Dhaka Zoo and stained with H\&E. Scattered distributed granuloma was seen in the lungs tissue consisting of mononuclear cells and fibroblast (A, arrow head, 440x). Copious pink color serous exudate was seen in the distended and ruptured alveoli (B, arrow head, 220x). In general the lung alveoli were emphysematous (C, arrow head, 220x) and interstitial spaces were found to contain inflammatory deposits. Black color carbon particle was found to deposit in the cytoplasm of macrophages in the lungs (D, arrow head, 440x)

\section{Staining character}

Gram's and Leishman's staining were performed on smear of sample obtained from heart blood isolated pure culture and from broth and showed the presence of gram negative cocco-bacillary bipolar bacteria (Fig. 3B). Similar character was also observed with organism grown in various media and stained with Gram's and Leishman's stains. The bipolar nature and coccoid appearance of the organism was observed while the smear stained with Leishman's staining. 

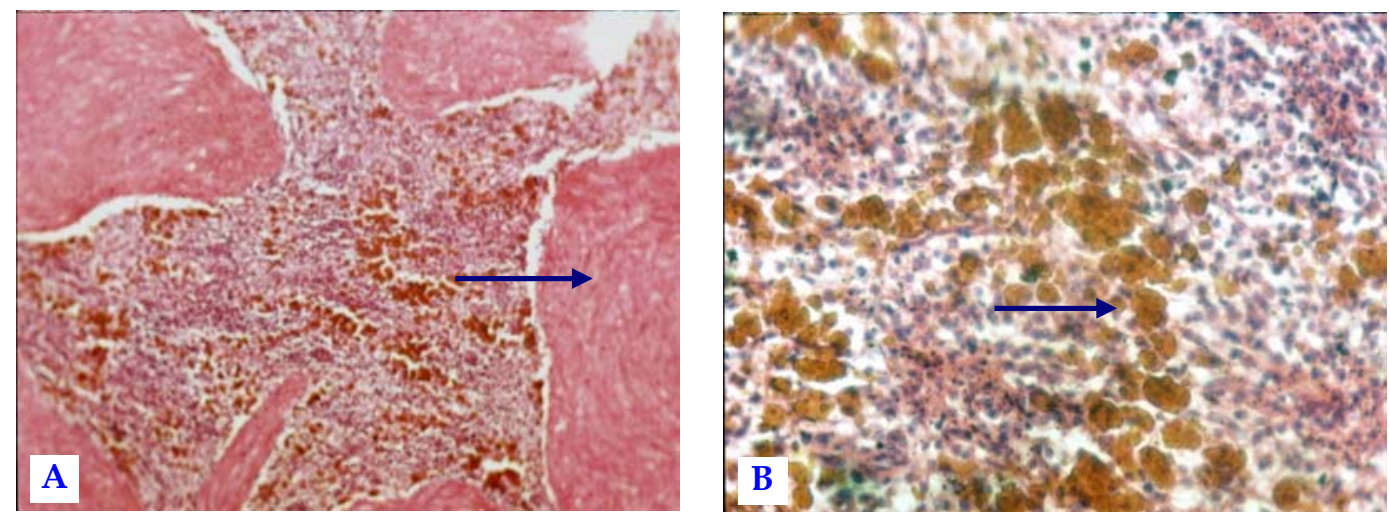

Fig. 2. Section of a spleen (Left) and lymphnode (right) of a dead zebra obtained from Dhaka Zoo and stained with $\mathrm{H}$ \& E. Lymphoid depletion was seen in the white pulp of spleen resulting in the expansion of the splenic trabeculi (A, arroe head, 220x). Lymphoid depletion was also seen in the lymphnode. There was deposition of golden brown color hemosiderin pigment in the macrophages of spleen and lymphnode (B, arrow head, 440x)
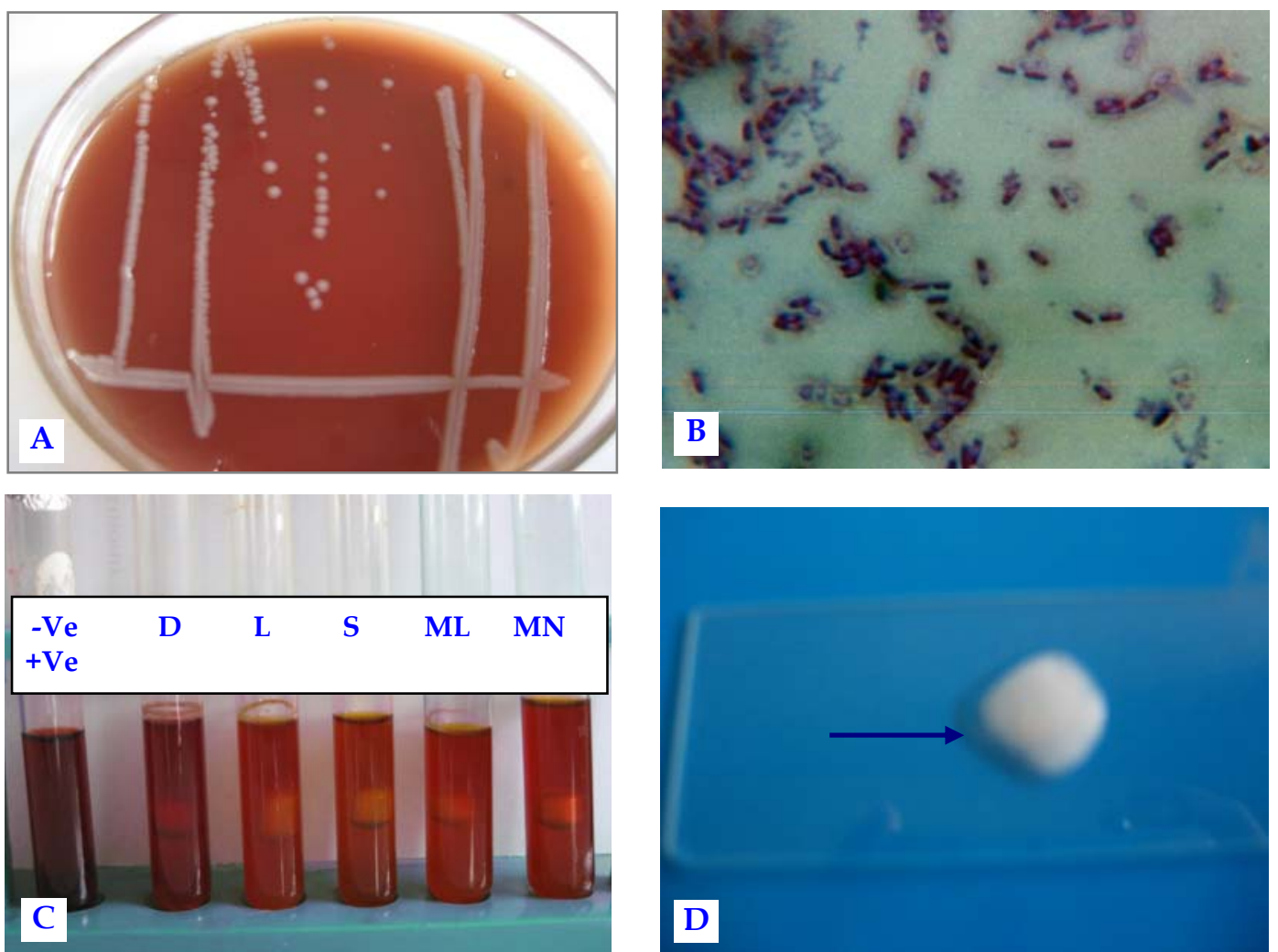

Fig. 3. White dew drop like (A) and grayish-yellow (arrow) non adherent colonies were seen on blood agar media. Isolated pure culture obtained from the thoracic fluid of a dead Zebra and stained with Gram's and Leishman's staining $(\mathrm{B} \times 1000)$ revealed gram negative, bipolar, capsulated (B, arrow head) coccobacilary organism. Results of sugar fermentation test (lower left, C) showed that the isolated pure culture ferment Dextrose (D), Lactose (L), Sucrose (S), Maltose (ML) and Manitol (MN) and only produce acid. Formation of bubbles (bottom right) using catalase reaction indicated that the organism is catalase positive and the organism is suggestive of pasteurella sp. 


\section{Biochemical characteristics}

Fermentation reaction with five basic sugars like dextrose, sucrose, mannitol, lactose and maltose, the organism ferments all sugars with the production of acid (Fig. 3C). In indol test, the appearance of red reaction is indicative of the positive test. Indol test was found to be negative and is suggestive for the isolates to be of Pasteurella sp. M-R test gives red ring for positive reactivity but it did develop yellow color product, indicated $\mathrm{M}-\mathrm{R}-\mathrm{Ve}$. V-P test procedure also produced negative results. Pasteurella $s p$. always give -Ve test results for MR and VP test. In catalase activity test, the organism formed bubble, appeared positive to catalase and the test results is indicative of Pasteurella sp. (Fig. 3D).

Zoos are having wide biodiversity and potential source of recreation as well as source of emerging and re-emerging infectious diseases. It has public health concern and provides innocent reaction among other important implications of merriment (Tribe, 2004). Around 150 infectious diseases are listed in recent years originated from wild life population both in captivity and free ranges (OIE, 2007). Among zoo animals zebras are occupied at the center of all zoo collection. Dhaka Zoo also having considerable number of zebra, collecting from African territory. There was frequent illness of zebras of Dhaka Zoo and this study was, therefore, conducted to investigate the diseases of zebra (Equs burchellii) at Dhaka Zoo.

\section{DISCUSSION}

Two zebra at Dhaka Zoo fallen ill and died later on during the mid June 2010 and two others were getting ill. Systemic postmortem examination was carried out and the most important necropsy findings observed were generalized congestion, congested and consolidated lungs, hydrothorax, and hydropericardium. Lightly grey color focal necrotic area was seen on the surface. The gross lesions described in this study were almost similar to those described earlier (Okoh, 1980 and Harish, 2005) and commonly observed in pasteurella induce septecemia. So the lesions were assumed to be the case of pasteurellosis. Therefore, systemic histopathological examination was carried out.

The striking histopathological findings observed were pulmonary emphysema, interstitial pneumonia, fibrinous pneumonia, thrombosis in the vasculature of lungs and seropurulent bronchitis. This finding was similar to those described by Gazquez et al. (2001); Jones et al. (1999) and Tehrani et al. (2004); in pasteurella induce pneumonia. Granulomatous lesions was also found in lung and the lesion was confused with bovine tuberculosis but in Ziehl-Neelseen method of staining acid fast organism was not detected. So the granulomatous lesions developed in lung tissue could be due to some other reason and has not been identified in this study. Another important finding was hemosiderosis and anthracosis. There were deposition of high amount of hemosiderin in spleen, lymphnode and lungs. Hemochromatosis has previously been reported in the horse, rhinoceros, and in one Brazilian tapir (Bonar et al., 2006). The deposition of higher amount of hemosiderin pigment in the spleen, lymphnode and lungs could be due to increase destruction of red blood cells but the reason could not identified. There were 
deposition of carbon particle (black pigment) in lungs and regional lymphnode. This histopathological lesion described in the present study were almost similar to those described by Sujatha et al. (2007); Jubb et al. (1993); Jones et al. (1999); Morali Manohar et al. (2006) and Yu-Tong Yao (1984). Dhaka Zoo is located in a point around which thousands of vehicles were passing through and the anthracosis in zebra as seen could be due to the inhalation of smoke. People dwelling in Dhaka city perhaps suffering with same ailment which is our apprehension. Another anticipation might be drown from this study is that anthracosis may predispose a few respiratory illness along with the development of carcinogenesis. Anthracosis in animal, indicate carbon particle and dust pollution in environment that needs a special attention. However, most of our attention was diverted to find bacteriological agent with the illness of zebra and bacteriological investigation was carried out.

In this study identification of pasteurella organism was done which has important role as primary or opportunistic, mainly respiratory pathogens in domestic and wild animals (Dousse, 2009). Identification of Pasteurella sp. was done on the basis of cultural and staining morphology and results of biochemical tests. The colony of the organism on blood agar appeared as round, slightly raised, smooth, shiny grayish-yellow in color, and non adherent. Hemolysis was not seen in blood agar. On MacConkey agar plate, bacteria produce small pink colour colony. The organisms were demonstrated as Gram-negative coccobacilli and bipolar capsulated rods using Gram's and Leishman's stains respectively (Muhsin et al., 1977; Choudhury et al., 1985; Prasad et al., 1997 and Linda and Schlater, 1989). Results of bacteriological tests showed that the organism is Pasteurella sp. The Pasteurella sp. commonly seen in animals are P. aerogenes, P. multocida and P. hemolytica. Biochemical tests are commonly used to identify the species of Pasteurella. On the basis of biochemical tests an organism would consider as $P$. aerogenes if the organism ferment glucoes, sucrose, lactose, maltose, manitol and lacks of indol production (Linda and Schlater, 1989). P. multocida ferment glucose, sucrose and manitol but do not ferment lactose and maltose. P. multocida yield positive indol reaction (Okoh, 1980). P. hemolytica lyse blood on blood agar, ferment glucose, sucrose, lactose, maltose, manitol and negative in indol reaction (Wessman, 1968). The results of biochemical tests could not correlate entirely with any property of $P$. multocida, P. hemolytica or P. aerogenes. However, most of the findings of this study supported the idea that the organism could be $P$. aerogenes. The animals were collected from African territory. Intense serological tests using specific monoclonal antibody or genetic analysis could be of value to identify the species of Pasteurella involved.

\section{CONCLUSION}

The present research work was conducted to investigate the cause of death of zebra (Equus burchelli) at Dhaka Zoo. For this purpose different samples were obtained and investigated histopathologically and bacteriologically. Histopathological findings revealed pulmonary emphysema, interstitial pneumonia, bronchitis, pulmonary thrombosis, exudates in lung alveoli and granulomatous lesions in lungs. Another 
important findings were hemosiderosis and anthracosis. Prevalance of anthracosis in zebra of Dhaka Zoo might be due to excessive smokes and dust in the air in and around the zoo captivity. The prevalence of anthracosis due to burning oil from unfil cars and industrial smokes in zebra indicate that human being living at Dhaka city might have the same lesion in their lungs.

Thoracic fluid, heart fluid, and abdominal fluid, cardiac fluid were investigated for bacteriological study using different culture media including nutrient agar, blood agar, MacConkey agar, Sugar fermentation test and other biochemical reaction. Isolated colonies obtained from these medium and stained with Grams and Leishman's stain showed Gram negative bi-polar and coco-bacilli organism characteristics of Pasteurella sp. Biochemical properties of the isolate was studied by sugar fermentation tests, indol test, methyl red and Voges-Proskauer tests. Enzymatic activity test such as catalase test was also performed. The reactions appeared similar to that of pasteurellaceae, may either be $P$. multocida, $P$. hemolytica, or $P$. aerogenes. This is the first report about the characterization of pasteurella isolated from zebra (Equus burchelli) at Dhaka Zoo. However, it was not clear whether the pasteurella isolated was belonging to any specific species. Therefore it needs further investigation at genetic level to confirm the bacterial species involved with this malady.

\section{REFERENCES}

Anon: http:/en.Wikipedia.Org/wiki/Dhaka Zoo Retrieved, 2009.

Bonar, C. J., Trupkiewicz, J. G., Toddes, B. L. and Ewandowski, A. H. 2006. Iron storage disease in tapirs. Journal of Zoo Wildlife Med, Official Publication of American, Association of Zoo Veterinarians. 37(1): 4952.

Choudhury, R. A., Amin, N. M., Rahman, A. and Ali, M. R. 1985. Investigation of natural outbreak of fowl cholera. Bangl. Vet. J., 19: 49-56.

Dhand, -N-K. 2004. An outbreak of pasteurellosis in small ruminants in Punjab. J. Res. Punjab Agri. Univer., 41(1): 147-149.

Dousse, F., Thomann, A., Brodard, I., Korczak, B. M., Schlatter, Y., Kuhnert, P., Miserez, R., Frey, J. 2009. Routine phenotypic identification of bacterial species of the family Pasteurellaceae isolated from animals. J. Vet. Diagn Invest., 20(6): 716-24.

Gazquez, -A., Redondo, -E., Martinez, -S., Gomez, -L. 2001. Ultrastructural study of ovine pulmonary pasteurellosis: Involvement of neutrophils and macrophages. Histology-and Histopathology, 16(3): 855-860.

Harish, -B-R. 2005. An outbreak of swine pasteurellosis and its pathology. Indian J. Vet. Path., 29(2): 129-130.

http:/ / www.natureartists.com/zebras.asp. Retrieved, 2008-10-10.

John, G. Kurt, B. Paul, S. Jeff, R. Phillip T. R. 1984. Hemosiderosis in lemurs. Res. Center Zoo Biology, 3(3): 255-265.

Jones, T. C., Hunt R. D. and King, N. W. 1977. Diseases caused by fungi in the veterinary pathology $6^{\text {th }}$ edn., Williams and WILKINS. 8: 50522. 
Jones, T. Carlyle, Hunt, Duncan, R., King, N. 1999. Veterinary Pathology, 6th ed., Williams \& Wilkins, pp. 957, J76V, C., 14(6).

Jubb, K. V. F., Kennedy, P. and Palmer, N. 1993. Pathology of domestic animals $4^{\text {th }}$ ed. Academic Press, San Diego, 641.

Kristine, M. Smith, Denise McAloose, Ann-Marie Torregrossa, Bonnie, Raphael, L., Paul, P. Calle, Robert, Moore, P., James, B. 2008. Hematologic iron analyze values as an indicator of hepatic hemosiderosis in callitrichidae. American J. Primatol., 70(7): 629-633.

Linda, R. Schlater, K. 1989. An acrogenic Pasteurella-like organism isolated from horses. J. Vet. Diagn Invest., 1: 3-5.

Luna, L. 1968. Manual of histologic staining methods of the armed forces institute of pathology. $3^{\text {rd }}$ ed. Mcgraw-hill.inc. Book Company, New York.

McClintock, Dorcas. 1976. "A Natural History of Zebras". Scribner's, New York. ISBN 0-684-14621-5.

Migliore, E., Serraino, C., Brignone, C., Ferrigno, D., Cardellicchio, A., Pomero, F., Castagna, E., Osenda, M., Fenoglio, L. 2009. Pasteurella multocida infection in a cirrhotic patient: case report, microbiological aspects and a review of literature. Adv. Med. Sci., 54(1): 109-12.

Motu, S. A. A. 2009. Pathplogical investigation of zoo collection with special reference to Tuberculosis and Mycosis. Department of Pathology, Bangladesh Agricultural University, Mymensingh, pp. 59.

Murali Manohar, B. 2006. Anthracosis of Leaf monkey (Presbytis pileatus). Tamilnadu J. Vet. Anim. Sci., 2: 112.

Mushin, R., Bock, R. and Abrams, M. 1977. Studies on Pasteurella gallinarum. Avian. Pathol., 415-423.

Office International Des Epizootic (OIE), 2007. Hemorrhagic Septicemia, pp. 1-3. The center for food security and public health, Collage of Veterinary Medicine, Iowa State University.

Okoh A. E. J. 1980. An outbreak of pasteurellosis in Kano Zoo, National Veterinary Research Institute, Diagnostic and Investigation Kano, Nigeria. J. Wildlife Diseases, 16(1): 140-160.

Prasad, V., Murthy. K. K. and Rao, T. V. J. 1997. In vitro antibiogram studies of E. coli in chickens. Indian. Vet. J., 76(7): 616-61.

Raghunathan, M. and Nareshwar, M. 2000. Zoo Education and Interpretation-Opportunities and Challenges Centre for Environment Education Ahmedabad, India, zooeducation@ceeindia.org

Sujatha K., Ch. Srilatha and Sasidhar Babu, N. 2007. Pulmonary anthracosis in large wild fields in vet brief , ZOOS' Print J., 22: 2902.

Tehrani, -A-A. 2004. Isolation and identification of Pasteurella haemolytica biotype A from sheep in Urmia, Iran. Iranian J. Vet. Res., 2004; 5(2): 105-109.

Tribe, A. 2004. 'Zoo tourism' in Wildlife Tourism: Impact management and planning. Higginbottom, Karen (ed), Common Ground Publishing, Altona, Victoria, 35-56.

Wessman, G. E. and Geraldine, H. 1968. Characterization of Pasteurella hemolytica Isolated from the Respiratory Tract of Cattle, Ames, Iowa Can. J. Comp. Med., vol. 32.

Yu-Tong, Y. 1984. Mineral dust in lungs with scar or scar cancer. Presented the meeting of the International Association of Pathologists, Atlanta, Georgia, from the Department of Pathology, National Taiwan University School of Medicine, Taiei Taiwan and the Department of Pathology, Mcgill University, Montreal, Quebec, Canada , Cancer, 54: 1814-1823. 